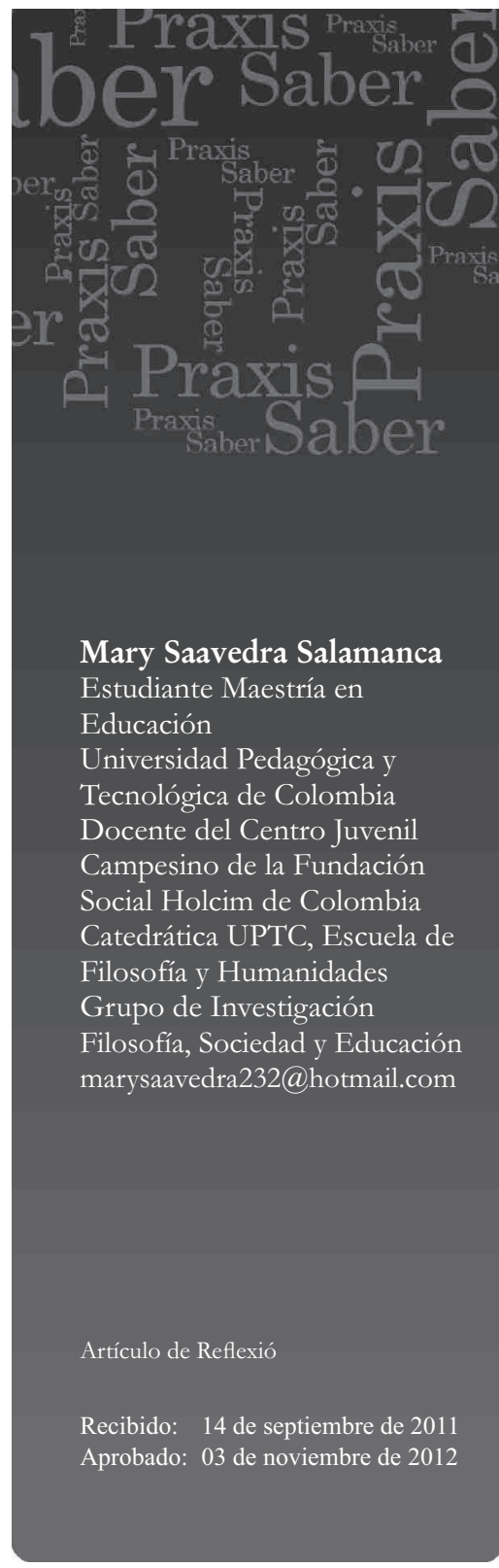

\title{
DEL AULA DE CLASE TRADICIONAL A LA COMUNIDAD DE INVESTIGACIÓN ${ }^{1}$
}

\section{Resumen}

Se aborda la relación entre la transformación de los modos de acción en el aula de clase tradicional y la posibilidad de realizar discusión filosófica en esta, en la que niños y jóvenes pueden desarrollar y fortalecer habilidades y destrezas de pensamiento que les permitan pensar por sí mismo de forma crítica y creativa. La perspectiva metodológica que se asume es cualitativa, y la recolección de la información se realizó a través de la Comunidad de investigación, estrategia teórico-metodológica sugerida por el programa Filosofía para Niños. Esta reflexión se realiza a partir de la experiencia pedagógica investigativa e innovadora de enseñar filosofía para niños en el colegio Centro Juvenil Campesino de la Fundación Social de Holcim Colombia,y también desde algunos referentes teóricos de la propuesta educativa de Filosofía para niños, referentes que han orientado el desarrollo de esta experiencia. En la parte final del texto se presentan los resultados de la experiencia y algunos testimonios sobre sus efectos en la comunidad educativa y en los sujetos involucrados en el proceso.

Palabras clave: Enseñanza de la filosofía, Filosofía para Niños, Comunidad de investigación, Discusión filosófica, Filosofía de la educación, Innovaciones pedagógicas.

\footnotetext{
1 Este trabajo hace parte del proyecto de investigación que se inicia para optar al título de Licenciado en Filosofía en el año 2009, proceso que ha contribuido a la práctica docente y a continuado en el proceso de investigación del trabajo de tesis de la Maestría en Educación en la Línea de Innovaciones Pedagógicas de la UPTC y del grupo de Investigación Filosofía, sociedad y educación en la línea Filosofía de la educación y enseñanza de la filosofía.
}
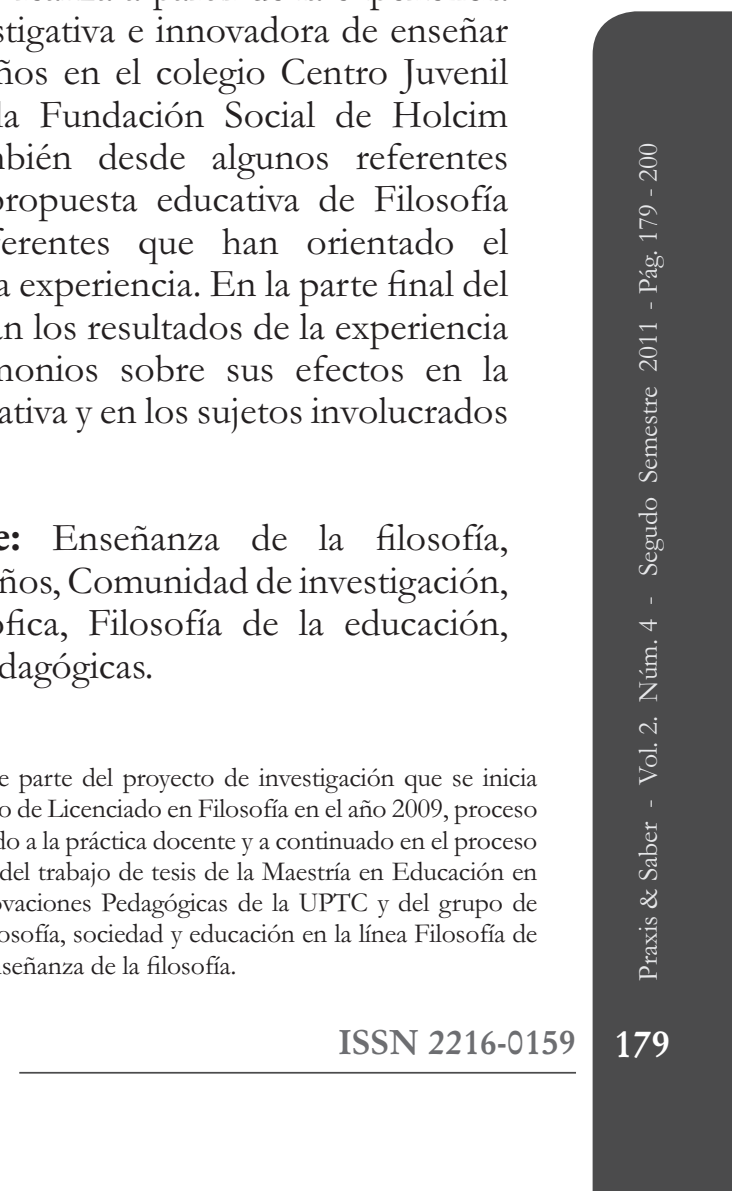


\section{Research as a Pedagogical Strategy a Step Towards Critical Pedagogies for the $21^{\text {St }}$ Century}

\section{Summary}

This research paper aims to promote the idea that the use of research in the fields of education and pedagogy is going to create a modification in these fields, as well as in the practical processes and actions that they have established. First of all, this paper provides a quick overview of the way in which education and pedagogy have been constituted in the Western, capitalist modern society; along with its paradigms, trends, concepts, methodological approaches, research areas and models. Next, this paper shows the way in which pedagogical thinking has been formed around research, that which gives rise to diverse methodological approaches which become the daily practice of educators. Finally, this paper closely examines one of these methodological lines, Research as a Pedagogical Strategy (Investigación como Estrategia Pedagógica - IEP), which emerged in the creation of the critical pedagogies developed in Latin American contexts, in dialogue with the emerging and dynamic approaches in different parts of the world.

Key words: Pedagogical research, educational paradigms, new capitalism, teacher.

\section{De la Salle de Classe Traditionnelle À la Communauté de Recherche}

\section{Résumé}

On aborde la relation entre la transformation des modes d'action dans la salle de classe traditionnelle et la possibilité d'y réaliser de la discussion philosophique; situation dans laquelle des enfants et des jeunes peuvent développer et fortifier des habilités et des compétences de pensée qui leur permettent de penser par eux-mêmes de manière critique et créative. La perspective méthodologique assumée est qualitative, et la récolte de données a été faite à travers la Communauté de recherche, une stratégie théorique-pratiquesuggérée par le programme de Philosophie pour Enfants. Cette réflexion est faite à partir de l'expérience pédagogique investigatrice et innovatrice d'enseigner de la philosophie aux enfants dans le collège Centro JuvenilCampesino* de la Fondation Sociale de Holcim Colombie, et aussi à partir de quelques référents théoriques de 
la proposition éducative de Philosophie pour enfants ; ceux-là ontorienté le développement de cette expérience. A la fin du texte on présente les résultats de l'expérience et quelques témoignages sur ses effets dans la communauté éducative et chez les sujets involucrés dans le processus.

Mots clés: Enseignement de la philosophie, Philosophie pour Enfants, Communauté de recherche, Discussion philosophique, Philosophie de l'éducation, Innovations pédagogiques.

\section{Da Sala de Aula Tradicional à Comunidade de Pesquisa}

\section{Resumo}

Desenvolve-se a relação entre a transformação dos modos de ação na sala de aula tradicional e a possibilidade de fazer discussão filosófica nela, onde crianças e jovens podem desenvolver e fortalecer habilidades e destrezas de pensamento que lhes permitam pensar por eles mesmos de forma crítica e criativa. Assume-se o enfoque metodológico qualitativo, e desta forma a coleta de informação se fez através da comunidade de pesquisa; estratégia teórico-metodológica sugerida pelo Programa Filosofia para Crianças. Faz-se a reflexão a partir da experiência pedagógica investigativa e inovadora de ensinar filosofia para crianças do colégio Centro Juvenil Campesino da Fundação Social Holcim Colômbia, e alem disso desde alguns referenciais teóricos da proposta educacional de Filosofia para Crianças. No final do texto apresentam-se os resultados da experiência e algumas testemunhas sobre os seus efeitos na comunidade educacional e nos sujeitos envolvidos no processo.

Palavras chave: Ensino da filosofia, filosofia para crianças, comunidade de pesquisa, discussão filosófica, filosofia da educação, inovações pedagógicas.

...no les haremos un gran favor a los niños invitados a pintar una acuarela de la filosofía si nos les permitimos que entren con sus telas, sus pinturas, sus pinceles y, sobre todo, su propio modo de pintar y concebir la pintura. Claro, puede que les guste y se diviertan con nuestros pinceles y nuestras pinturas. Incluso si las utilizan desde pequeñitos puede ser que sientan que las necesitan y se vuelvan muy expertos en ellas. Pero, probablemente, en algún momento sentirán que esos instrumentos y esos diseños les son extraños, que al adoptarlos están confinados a creaciones de otros, que los enajenan y no les sirven para expresarse a sí mismos.

Walter Omar Kohan

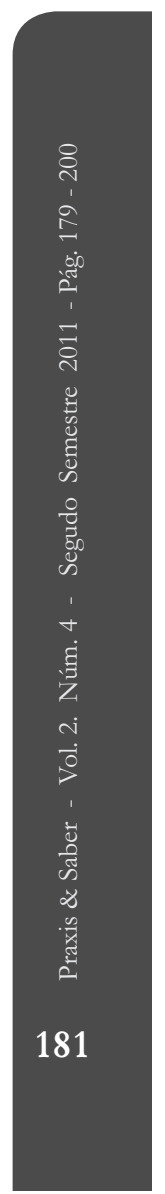




\section{Introducción}

En la decisión de emprender el proceso de investigación para optar al título de Licenciada en Filosofía siempre estuvo presente la inquietud por las preguntas qué, cómo y para qué enseñar filosofía, fuente de discusión entre compañeros y amigos de la carrera. Tras la práctica docente se dio la posibilidad de enseñar filosofía en educación básica y media, una oportunidad que articuló estas preguntas con el objetivo del trabajo de investigación, orientado en aquel momento al diseño de una propuesta curricular que permitirá enseñar filosofía de forma estructurada. Este proceso permitió, por una parte, diagnosticar el estado de la enseñanza de la filosofía en la institución educativa, y, por otra, reconocer los fundamentos de la propuesta educativa de Filosofía para Niños, ejercicio de indagación que contribuyó a la formación, reflexión y práctica docente.

Al no ser la propuesta curricular un esquema determinado y acabado, el proyecto de investigación se orientó a revisar y evaluar los procesos, y a transformar y a hacer adaptaciones que en conjunto beneficiaran la enseñanza de la filosofía y, con ello, la transformación del aula de clase tradicional en comunidad de investigación, como un ambiente propicio para el desarrollo de habilidades y destrezas de pensamiento que permitiera a niños y jóvenes pensar por sí mismos de forma reflexiva y crítica. Hoy, estas acciones orientan el trabajo de tesis ${ }^{2}$ que se adelanta en la Maestría en Educación con la Línea de Innovaciones Pedagógicas.

Bajo estas consideraciones, el presente artículo tiene el propósito de presentar, desde la práctica de enseñar filosofía en el colegio Centro Juvenil Campesino, de la Fundación Social de Holcim Colombia ${ }^{3}$, la relación entre la transformación de los modos y formas de acción del aula de clase tradicional y la posibilidad de realizar discusión filosófica

2 El trabajo de investigación que se adelanta sobre la enseñanza de la filosofía en doce instituciones educativas de la provincia de Sugamuxi recoge las reflexiones sobre la práctica docente como fundamento que orienta tanto el análisis de los modos y formas de enseñar filosofía en estas instituciones, como la posibilidad de contribuir a la transformación de las aulas de clase en comunidades de investigación.

3 La Fundación Social de Holcim Colombia es la entidad que encabeza la tarea de ejecutar la Política de Responsabilidad Social de la cementera Holcim. El Centro Juvenil Campesino (CJC) es el programa de educación por medio del cual se desarrolla el proyecto de Educación y Medio Ambiente de la Fundación. El CJC está ubicado en el municipio de Nobsa, Boyacá; es una institución educativa de carácter privado que tiene como objetivo brindar educación básica y media a niños y jóvenes del sector rural de once municipios de la zona de influencia de la cementera Holcim. 
con niños y jóvenes de diferentes edades. En su primera parte, el artículo indica los fundamentos de la propuesta educativa de Filosofía para Niños, que fueron tomados como punto de análisis y reflexión sobre la práctica docente, y luego presenta la experiencia de enseñar filosofía desde la transformación del aula de clase tradicional en comunidad de investigación.

\section{Enseñanza de la filosofía y Filosofía para Niños (FpN)}

La preocupación por la formación de las nuevas generaciones y por el papel de estas en la transformación social ha impulsado en los últimos tiempos la revisión de propuestas educativas que plantean enseñar filosofía a niños y jóvenes de diferentes edades con el objetivo de generar e incentivar la reflexión, la crítica y el análisis de situaciones problemáticas del entorno. La propuesta conocida como Filosofía para Niños $(\mathrm{FpN})$ es, posiblemente, la de mayor repercusión e incidencia en la discusión que se ha originado en diferentes países sobre la enseñanza de la filosofía y su función en la formación ética y política de las nuevas generaciones; por esta razón, se hace necesario revisar algunos de los fundamentos que justifican esta propuesta, reflexionar en torno a la forma como se ha enseñado filosofía y, a su vez, hacer referencia a los elementos que potencialmente pueden adoptarse para generar una propuesta innovadora en otros escenarios académicos.

El proyecto y programa Filosofía para Niños aparece en Estados Unidos a finales de la década de los sesenta del sigo pasado, inspirado por el profesor Matthew Lipman, de la Universidad de Montclair. Desde su origen, se reconoce como una propuesta educativa que permite acercar a niños y jóvenes al mundo de la filosofía; que propicia el desarrollo del pensamiento, la conceptualización y las actitudes responsables desde el punto de vista ético y político, y que contribuye, desde la formación de las nuevas generaciones, a la construcción de una sociedad más libre y solidaria.

Filosofía para Niños ha originado diversas reflexiones en torno a la relación entre filosofía y educación, y en particular sobre el papel de enseñar filosofía en las instituciones educativas. Las preguntas de qué, cómo y para qué enseñar filosofía parecen encontrar en esta propuesta un marco de referencia, donde los fundamentos teóricos, las adaptaciones y aplicaciones que se han venido desarrollando se convierten para los docentes en fuertes argumentos que justifican iniciativas de transformación en la manera de enseñar filosofía y de concebir su función y utilidad en la 
formación de los estudiantes. Así, la enseñanza de la filosofía, que estaba dirigida exclusivamente para los grados décimo y once como una mirada general a la historia de la filosofía, hoy es pensada para niños y jóvenes de otros grados y de diferentes edades, como un ejercicio para el desarrollo de destrezas y habilidades de pensamiento que le permiten al estudiante pensar por sí mismo.

El profesor Matthew Lipman considera que el objetivo de la FpN no tiene lugar ni repercusión en una educación dedicada exclusivamente a la memorización, y señala como una de las mayores falencias de la educación tradicional la falta de formación para lograr personas razonables. La sociedad necesita personas razonables capaces de transformar el presente en función de un futuro mejor; no se puede pretender generar algún tipo de cambio cuando los procesos de enseñanza y de aprendizaje de las nuevas generaciones están basados en la memorización y en la repetición mecánica de contenidos. La educación tradicional muestra a los estudiantes memorizando y aprendiéndose los contenidos de las asignaturas, mientras fracasan cuando llega la hora de resolver problemas de la vida cotidiana (Lago, 2006). Luego, la transformación en función de lograr personas razonables que piensen por sí mismas depende de que la forma como se concibe la enseñanza de la filosofía y el ambiente que se genera en el aula de clase impliquen acciones que deben estar siempre dirigidas a valorar las inquietudes y preguntas de los estudiantes en función de generar cuestionamientos y reflexiones que generen nuevas y mejores preguntas.

Para comprender la posición del profesor Matthew Lipman frente a las falencias de la educación tradicional y a la posibilidad de la propuesta educativa de $\mathrm{FpN}$ es necesario hacer mención a la diferencia entre práctica normal y práctica crítica de la educación, que presenta en su libro Pensamiento complejo y educación ${ }^{4}$; diferencia que permite visualizar, en uno y otro tipo de educación, la forma como se concibe la filosofía y la enseñanza de la filosofía en las instituciones educativas. Lipman considera como supuestos de la práctica normal de la educación:

- La educación consiste en la transmisión del conocimiento de aquellos que saben a aquellos que no saben.

- El conocimiento es sobre el mundo, y nuestro conocimiento sobre el mundo es preciso, inequívoco y no-misterioso.

4 En el libro Pensamiento complejo y educación, Matthew Lipman expone como problema de la educación tradicional la falta de estrategias pedagógicas que permitan la formación de estudiantes más reflexivos y más racionales, capaces de adoptar una posición crítica frente a los problemas de su entorno. 
- El conocimiento se distribuye entre las disciplinas, que no se sobreponen y, juntas, abarcan todo el mundo por conocer.

- El profesor desempeña algún papel de tipo autoritario en el proceso educativo, y espera que los estudiantes conozcan lo que él conoce.

- Los estudiantes adquieren el conocimiento mediante la absorción de datos e información; una mente educada es una mente bien abarrotada (1998, p. 55).

Cabe señalar que cada uno de estos supuestos bien podría ser parte de las características que definen la educación tradicional, y, por supuesto, de la manera como se da allí la enseñanza de la filosofía: una asignatura pensada para presentar la historia de la filosofía, concebida como un conjunto de obras filosóficas que deben ser divulgadas por los docentes y memorizadas por los estudiantes, con el único objetivo de ser replicadas en exámenes y pruebas externas. En tanto el estudiante memorice y recite la información, se cree que aprende y adquiere el ejercicio filosófico realizado, en otros tiempos, por estos pensadores. A partir esta concepción, la enseñanza de la filosofía replica los supuestos de la educación tradicional, considerando que la memorización de algunos datos de la historia de la filosofía, muchas veces presentada de forma desfragmentada; por lo tanto, no beneficia la comprensión que pueden lograr los niños y jóvenes sobre la dimensión filosófica de su propia experiencia y, aún menos, sobre los problemas filosóficos que se presentan en la historia. Esta situación contribuye y otorga funciones a la enseñanza de la filosofía que deterioran la posibilidad de vincular, desde el ejercicio filosófico en el aula de clase, la tradición filosófica con la formación de estudiantes razonables que puedan pensar por sí mismos sobre lo que les interesa y les genera inquietud.

Algunos la aprecian en la medida en que mejora las habilidades de razonamiento; otros la admiran porque los alumnos disfrutan con ella por sí misma, más que por sus calificaciones o porque sea importante para sus aspiraciones vocacionales. Algunos la ven como el tronco central de la escuela elemental y secundaria, a partir del cual pueden ramificarse las asignaturas especializadas. Otros, como saludable prevención contra las drogas o el alcoholismo (Lipman, 2002, p. 37).

La falta de claridad respecto a la función de enseñar filosofía en las instituciones educativas puede contribuir a justificar iniciativas de reducir, incluso suprimir, el espacio en los planes de estudio de educación básica y media. Una asignatura que, a diferencia de las áreas básicas, pareciera no tener espacios de concreción y, aún menos, claridad sobre la manera de incidir en la formación de los estudiantes. Por esta razón, la 
implementación y adaptación de la propuesta educativa de FpN no solo debe posibilitar que niños y jóvenes puedan pensar por sí mismos desde la reflexión e investigación filosófica, sino también aportar a la revisión y trasformación de las formas de enseñar filosofía en los grados décimo y once. Alternativa que tiene como principio la relación de preguntas e inquietudes de los estudiantes con las problemáticas y cuestiones de la tradición filosófica. Las referencias históricas, el análisis de los textos y la relación con la vida cotidiana del estudiante deben ser orientados al desarrollo de habilidades y destrezas de pensamiento que les permita comprender su relación con el mundo, con los demás y consigo mismos.

En este sentido, los supuestos de la práctica crítica que expone el profesor Matthew Lipman sobre la educación podrían estar acordes con los objetivos que persigue la propuesta educativa de $\mathrm{FpN}$, en cuanto se puede desde allí concebir otras maneras de enseñar filosofía y ver su función en el campo de la educación. Lipman afirma:

- La educación es el objetivo de la participación en una comunidad de indagación guiada por el profesor, entre cuyas metas están la pretensión de comprensión y de buen juicio.

- Se anima a los estudiantes a pensar sobre el mundo cuando nuestro conocimiento sobre él se les revela ambiguo, equívoco y misterioso.

- Las disciplinas en el interior de las escuelas se generan por procesos indagativos, pueden yuxtaponerse entre ellas y, además, no son exhaustivas en relación con su respectiva área de conocimiento, que es problemática.

- El profesor adopta una posición de falibilidad (aquel que admite estar equivocado), más que de autoritarismo.

- Se espera que los estudiantes sean reflexivos y pensantes, que vayan incrementando su capacidad de razonabilidad y de juicio.

- El foco del proceso educativo no es la adquisición de información, sino la indagación de las relaciones que existen en la materia bajo la investigación (1998, p. 56).

Bajo estas acciones, la enseñanza de la filosofía, así como de otras asignaturas, está orientada a mantener un vínculo permanente entre lo que pasa en el aula de clase y la vida cotidiana del estudiante; no puede ser, entonces, una asignatura más del plan de estudios, es un ejercicio que convoca el diálogo y la discusión como medios para fortalecer habilidades de pensamiento y razonamiento sobre la vida misma. Luego, el docente de filosofía debe visibilizar la conexión entre las cuestiones filosóficas 
y las preguntas e inquietudes de los estudiantes, aquellas preguntas que surgen no solo de sí mismos, sino también sobre el mundo y la sociedad en que viven; no sin antes privilegiar y celebrar acciones que con el tiempo se consolidan como hábitos propios de la actitud filosófica: dar y solicitar argumentos, problematizar y cuestionar lo evidente, hacer preguntas y contemplar en una discusión alternativas de análisis y solución, son algunas de las acciones que transforman la visión tradicional de la enseñanza de la filosofía e inciden en la posibilidad de construir y asumir actitudes filosóficas sobre los problemas de la cotidianidad. En este sentido, hablar de enseñar filosofía es hablar de un espacio propicio para el diálogo y la discusión filosófica, allí donde estudiantes y docentes pueden realizar un trabajo colaborativo que beneficie la comprensión de los problemas de la filosofía y, a su vez, influya en la manera como se asumen y resuelven los problemas que se presentan en la vida cotidiana.

... si la filosofía quiere ser útil en la transformación de la sociedad, si la filosofía quiere dar satisfacción a la vinculación con la educación humanizadora y afrontar los problemas y retos de la nueva sociedad [...] debe, desde un planteamiento total e integrado, hacer frente a los problemas que hoy en día surgen, preparándonos para afrontarlos de una manera crítica e innovadora, y para no contentarnos únicamente con lo dado, sino buscar más allá de lo evidente e investigar por el sentido profundo de la realidad, el mundo y de los cambios que en el suceden (Lago, 2006, p. 51).

La posibilidad de incidir en la transformación de la sociedad depende de la forma como se presenta la filosofía a los estudiantes, y de la relación que estos puedan encontrar con su experiencia. Al respecto cabe señalar que la enseñanza de la filosofía puede estar dedicada, por una parte, a la presentación de la cultura filosófica, como el recuento histórico del pensamiento de los filósofos, y por otra, a la realización de ejercicios filosóficos que pueden ser orientados a la acción de filosofar (Benítez, 2000). En la enseñanza de la filosofía, vista desde los supuestos de la práctica crítica de la educación, no es posible privilegiar o excluir una u otra forma, sino filosofar, es decir, pensar de forma reflexiva y crítica la cultura filosófica; un ejercicio que se beneficia de manera recíproca, en tanto el filosofar mejora la comprensión de la filosofía, y esta, a su vez, incide en la calidad del razonamiento de los estudiantes.

... el problema de la historia de la filosofía, al aproximar la reconstrucción racional a la historia es darse cuenta de que, por su peculiar ubicación crítica, los contenidos filosóficos requieren, además del tratamiento historiográfico, de un enfoque filosófico que nos permita ordenarlos, 
analizar sus contenidos, apreciar su desarrollo, en una palabra, darle sentido al inmenso caudal filosófico (Benítez, 2000, p. 37).

La enseñanza de la filosofía, al priorizar la discusión filosófica, aplica un enfoque orientado a desarrollar la capacidad de filosofar en los estudiantes; una actividad personal, pero no individual, en cuanto se necesita espacios de discusión y debate con otros. Lipman afirma: "La discusión agudiza las habilidades de razonamiento y de investigación en los niños como ninguna otra cosa puede hacerlo" (2002, p. 47). De esta manera, los estudiantes logran problematizar, conceptualizar y argumentar en una discusión filosófica que puede trabajar en todo momento las problemáticas que generan inquietud e incertidumbre en los estudiantes; la relación con las cuestiones filosóficas depende de la orientación del docente, quien puede decidir entre hacer una cátedra de filosofía o invitar e incentivar a los estudiantes a indagar e investigar sobre todo aquello que les resulta enigmático.

Los profesores de filosofía suelen hacer preguntas que los estudiantes pueden responder sin ningún tipo de apasionamiento, sin generación de dudas o perplejidades y sin que genere pensamiento auténtico, ya que el proceso suele ser mecánico y constreñidor. En cambio hay ocasiones en que empieza una indagación porque se ha hallado alguna aberración, alguna discrepancia, algo que se da por sentado captura nuestro interés y pide nuestra reflexión e investigación (Lipman, 1998, p. 58).

Bajo estas consideraciones, los docentes que creen en la posibilidad de lograr algún aporte a la transformación de la sociedad no solo deben llevar estas acciones al aula de clase, sino también, y en función de transformar sus prácticas, tomar posición crítica frente a los supuestos de la educación tradicional, analizar y estudiar el sentido y la pertinencia de los procesos de enseñanza y de aprendizaje, los objetivos que persigue y el tipo de sujeto que desean formar. Así, los alcances de propuestas educativas como FpN y de otras acciones innovadoras logran trascender el aula de clase y contribuir a la formación de una actitud reflexiva y crítica que cuestiona y problematiza la realidad, que busca alternativas de análisis y solución, que conceptualiza y argumenta de manera racional actitudes y formas de pensar, en fin, acciones que terminan cultivando una actitud filosófica expresada en el trabajo de indagación colaborativa entre docente y estudiantes.

Para finalizar este apartado, cabe señalar que la reflexión sobre la función de la enseñanza de la filosofía que aquí se presenta es resultado de la revisión inicial sobre los fundamentos de la propuesta educativa de FpN; revisión que parte del proceso de investigación que se inicia no solo para 
optar al título de Licenciada en Filosofía, sino también como proceso de actualización para ejercer como docente del área en el colegio Centro Juvenil Campesino, de la Fundación Social de Holcim Colombia; hecho que permitió replantear las preguntas de qué, cómo y para qué enseñar filosofía y considerar otras formas y modos de acción diferentes a los expuestos por la educación tradicional, con el fin de orientar el proyecto educativo de enseñar filosofía desde el grado sexto en adelante. En este sentido, la primera revisión, que provoca cuestionamiento e incertidumbre, es dirigida a buscar en la propuesta de $\mathrm{FpN}$ principios comunes que bajo las características de la población estudiantil y del proyecto institucional permitiera, por un lado, desarrollar destrezas y habilidades de pensamiento en los estudiantes en virtud de lograr el pensar por sí mismo de forma crítica y creativa, y por otro, brindar desde el ejercicio particular del área elementos que contribuyeran a denotar la función de la enseñanza de la filosofía en los procesos de formación de los niños y jóvenes de la institución educativa.

\section{La discusión filosófica en la comunidad de investigación como principio para la enseñanza de la filosofía}

Como se ha dicho, el profesor Matthew Lipman parte de considerar que las falencias del sistema educativo residen en la deficiencia para desarrollar destrezas y habilidades de pensamiento que mejoren la capacidad de cuestionar de los estudiantes; se cree que la falta de reflexión sobre el propio pensamiento y sobre la forma de razonar interfiere en la capacidad de identificar y cuestionar la relación con la cultura, el contexto y la noción misma de la persona que piensa. En este sentido, la enseñanza de la filosofía tiene como objetivo lograr que los estudiantes piensen por sí mismos a partir de la reflexión sobre su propio pensamiento, sobre cómo se piensa y sobre los razonamientos que se utilizan para deducir conclusiones o presentar soluciones a problemáticas en discusión.

Lago, al describir la función de la filosofía, hace referencia a los objetivos que puede desarrollar respecto a las falencias de la educación:

... la filosofía como disciplina [...] desarrolla las habilidades de razonamiento y pensamiento y de potenciar y perfeccionar las destrezas y las capacidades de los niños y niñas, y adaptándola a los distintos niveles educativos y de aprendizaje, se podrá lograr así el objetivo de introducir en el sistema educativo el pensar sobre el pensamiento y lograr desarrollar la capacidad de filosofar sobre la propia vida y sobre el tipo de persona que se es y se quiere llegar a ser (2006, p. 55). 
La discusión filosófica se convierte para la enseñanza de la filosofía en un mecanismo que incide en la manera como los estudiantes pueden pensar sobre su propio pensamiento. Las destrezas y habilidades de pensamiento y razonamiento son solo posibles de adquirir y fortalecer por medio de la discusión y la deliberación con otros. "El pensamiento es el diálogo interiorizado, pensar es hablar uno consigo mismo, luego, mejorando la calidad del diálogo, se mejora la calidad del pensamiento" (Lago, 2006, p. 55).

Favorecer la relación del lenguaje y el pensamiento en la discusión filosófica depende de la transformación del aula de clase tradicional en comunidad de investigación, donde los estudiantes puedan expresar sus puntos de vista, contar historias, hacer preguntas, comentar inquietudes $\mathrm{y}$, sobre todo, conversar y dialogar sobre lo que les genera inquietud e incertidumbre.

La comunidad de investigación no tiene una estructura definida y determinada; por la misma naturaleza de la discusión filosófica, está en constante evolución, cambio y adaptación al entorno, a las circunstancias y al contexto (Lago, 2006), y rompe con la estructura fija y determinada del aula de clase tradicional, cambia las filas por las mesas redondas y los trabajos en grupo, permite ir de la prohibición de mirarse a la posibilidad de reconocer y construir con el otro, y facilita pasar del silencio, la aceptación y la tolerancia a la discusión filosófica, en la cual la mejor forma de respetar al otro implica tomar sus puntos de vista para generar debate, con el firme convencimiento de que él, al igual que otros, puede tener en parte razón sobre lo que se dice. Las múltiples relaciones que se originan en torno a la discusión filosófica son resultado de las acciones no siempre condicionadas por el docente; con el tiempo, la posición de los estudiantes frente a las intervenciones suscitadas en la comunidad de investigación pasa de ser una norma externa a un hábito de los participantes.

... niños escuchándose y construyendo ideas unos con otros; aportando y analizando razones en sustento de los argumentos propuestos; ayudándose unos a otros a formular preguntas y a ampliar los puntos de vista; sustentando la hipótesis de alguien con un ejemplo; desafiando la de otro con un contraejemplo; dando tiempo y espacio para que las voces tímidas se expresen por sí mismas, y las voces agresivas se tornen más reflexivas y más consideradas (Sharp, 1995, p. 38).

En función de formar estos y otros hábitos, las actividades de la comunidad de investigación deben tener, en principio, el objetivo de incentivar 
la participación de los estudiantes, invitándolos a contar historias y anécdotas de la vida cotidiana, y luego, provocar el cuestionamiento, las preguntas y toda aquella acción que invite a la problematización de lo que se dice. "... se trata simplemente de animar al alumnado a que no saque conclusiones precipitadas, a que no se deje llevar por creencias aceptadas por la gente, a que no caiga en prejuicios y estereotipos, a que sea capaz de poner en duda sus propias ideas, ante la posibilidad de estar equivocado" (García, 2006, p. 149). La discusión filosófica se genera en la narración, pero se torna tal cuando los estudiantes adoptan el hábito de dar y pedir argumentos, de considerar otros puntos de vista y de reflexionar sobre su propio pensamiento.

De este modo, los docentes que construyen comunidades de investigación e incentivan el diálogo y la discusión filosófica con sus estudiantes pueden intervenir en la manera como construyen, organizan y expresan sus puntos de vista, $y$, al mismo tiempo, incidir en la forma como razonan y piensan. En principio esta será una tarea exclusiva del docente, pero poco a poco los estudiantes pueden ir haciendo las correcciones y observaciones necesarias para que sus intervenciones ganen en coherencia, sentido y pertinencia.

El profesor debe fomentar la cuestión, el criticismo, la discusión, actuando ahora como presidente, ahora como moderador, ahora como referencia, ahora como paladín de la invención, ahora como paladín de la tradición. Hay veces en que el profesor debe dar ánimos, pero otras en que debe dar consuelo [...] el profesor debe, en resumen, ser capaz de favorecer el diálogo, al menos hasta que la comunidad no necesite más apoyo o soporte externo (Lipman, 2005, p. 21).

Los alcances de la discusión filosófica dependen de la participación activa de los estudiantes en la comunidad de investigación, y del papel orientador del docente no solo sobre los modos y formas de intervenir, sino también sobre la manera de incentivar la revisión permanente de la forma como se piensa y razona. Por esta razón, las preguntas e inquietudes que se hacen los niños y jóvenes, no solo sobre sí mismos, sino también sobre el mundo y la sociedad en que viven, tienen un papel privilegiado en la discusión. No es posible generar autocorrección cuando no hay participación; las preguntas orientan el trabajo del docente y provocan al mismo tiempo la intervención de otros que piden razones de lo que se dice, de la forma como se piensa. Todo aquello que les interesa puede ser situación detonante para iniciar con otros la discusión filosófica donde 
todos puedan participar haciendo preguntas, solicitando argumentos, pidiendo explicaciones o, en ocasiones, precisando apuntes interesantes de algunas intervenciones. "El mundo que les rodea les provoca asombro y admiración, al tiempo que sorpresa y perplejidad, y les empuja a indagar en busca de respuestas a sus interrogantes" (García, 2006, p. 141).

La continua búsqueda de respuestas que caracteriza a los estudiantes puede encontrar en la comunidad de investigación un marco de referencia que da sentido y significado a aquello que les resulta enigmático o problemático, un lugar donde sus preguntas e inquietudes se tomen como fuente de discusión. En este lugar, la actitud que adopte el docente será ejemplo para los estudiantes; allí, el valor de las preguntas no radica en ofrecer respuestas concretas y fijas como verdades absolutas, sino en incentivar el cuestionamiento, la indagación y el surgimiento de otras preguntas. En palabras de Lipman: "El rol del docente de filosofía para niños no es responder preguntas, sino facilitar el surgimiento de preguntas por parte de los alumnos, así como la búsqueda de las respuestas" (2000, p. 28). Las preguntas llevan a adoptar una posición crítica y reflexiva sobre la realidad, despojando las falsas presunciones de sabiduría e indagando tras lo evidente. Por esto, se debe mantener el interés por preguntar, dado que lo que fortalece el razonamiento de los estudiantes es el ejercicio de construir preguntas que expresen lo que se quiere preguntar, y no el encontrar respuestas que no incentivan su curiosidad. Su importancia en la enseñanza de la filosofía depende entonces de la acción del docente, de que este tome en serio las preguntas de los estudiantes, trabaje en el aula de clase con ellas y, al mismo tiempo, exprese a sus estudiantes el hábito de preguntar, preguntado. García afirma que:

... el profesor de filosofía es más bien como un pez torpedo que a todo el mundo importuna con sus preguntas constantes, desmontando esas falsas seguridades en las que todos estamos instalados, y poniendo de manifiesto que en el fondo es más lo que ignoramos que lo que sabemos (2006, p. 143).

Provocar y fortalecer la discusión filosófica depende de mantener viva la capacidad de asombro de los estudiantes, como fuente de valiosas preguntas que promueven la participación en la comunidad de investigación, y con ello la posibilidad de indagar por los argumentos, el sentido de lo que se dice, la relación con otras preguntas y las razones de pensar de esta u otra manera. Este valioso ejercicio que permite mejorar la forma de pensar y razonar solo puede ser atendido por aquellos docentes que enseñan a pensar por sí mismos a sus estudiantes (Lipman, 2002). Se requiere 
docentes que, en lugar de creer saberlo todo, participen en la discusión filosófica con sus estudiantes, invitándolos a participar y construir puntos de vista provisionales que, opuestos a cualquier intención de instaurar verdades absolutas, permitan el cuestionamiento y la indagación filosófica.

La verdad sería una construcción comunitaria porque, gracias a diferentes puntos de vista y a la riqueza de la realidad, vamos elaborando una comprensión más profunda de lo que tenemos y, además, de que son posibles y válidas varias comprensiones. No todas, y en este sentido se supera la intersubjetividad: lo que digas contrástalo, convence con argumentos a los que te rodean. En una discusión no se admite aquello de Yo lo digo porque esa es mi opinión y punto (García, 2011, p. 14).

El valor de la pregunta se convierte en la única posibilidad de suscitar la discusión filosófica en la comunidad de investigación; sin ella, docentes y estudiantes vuelven a estar inmersos en la transmisión y memorización de la información. Su riqueza reside en potenciar la reflexión y el análisis sobre asuntos concebidos como verdades irrefutables, despertar la sospecha sobre lo que pasa inadvertido, incentivar la indagación y la curiosidad sobre todo aquello que genera interés e inquietud. La incertidumbre que origina y genera la pregunta en la discusión filosófica hace posible la construcción colaborativa y comunitaria de puntos de vista que favorecen la atención sobre alternativas de análisis y solución a las problemáticas que se presentan.

De forma paralela al ejercicio de dar valor a las preguntas e inquietudes de los estudiantes, es oportuno que el docente revise el tipo de material que en ocasiones utiliza para suscitar la discusión filosófica, la forma de presentarlo y el modo de usar ese material ${ }^{5}$ en la comunidad de investigación. Esa revisión debe permitirle orquestar lo que tiene con los objetivos que persigue con la enseñanza de la filosofía; priorizar por una didáctica del filosofar donde el valor de la discusión filosófica no está mediatizado exclusivamente por los libros, las películas y las imágenes, sino por el valor de la pregunta que permite al estudiante interrogarse a sí mismo y a los demás; un ejercicio cooperativo y colaborativo que contribuye a mejorar la

5 La propuesta educativa de Filosofía para Niños presenta una serie de novelas filosóficas acompañadas por manuales que pueden orientar la práctica docente, sin embargo, la posibilidad de implementar o adaptar la propuesta no está sujeta al uso de estos manuales, sino a la posibilidad de transformar las aulas de clase tradicional en comunidades de investigación que permitan a través de la discusión filosófica la formación niños y jóvenes más reflexivos y razonables. 
formulación de preguntas, la organización de los argumentos y la evaluación de las intervenciones en la discusión filosófica. En este sentido, el docente que desea influir en la transformación social debe recordar que toda decisión que se tome sobre la formación de los niños y jóvenes incidirá en la manera como ellos participen en la solución de situaciones problemáticas que afronte la sociedad. Seguramente, aquellos que hicieron parte de las comunidades de investigación asumirán una posición crítica que les permita contemplar con otros alternativas de análisis y solución.

\section{La transformación del aula de clase tradicional en el Centro Juvenil Campesino de la Fundación Social de Holcim Colombia}

Al enfatizar en la experiencia de enseñar filosofía en la educación básica y media se hace necesario señalar la relación de los estudiantes ${ }^{6}$ con el proceso que ha permitido ir del aula de clase tradicional a la comunidad de investigación. Así es posible dar cuenta de cómo la comunidad de investigación se adopta como marco de referencia que da sentido a la experiencia del estudiante, y a la vez se organiza como espacio de discusión y diálogo, donde pueden desarrollar habilidades y destrezas de pensamiento que les permita pensar por sí mismos con otros, de forma reflexiva y crítica. Para esto, la siguiente sección describe, por una parte, el escenario académico bajo el cual se propone la enseñanza de la filosofía desde el grado sexto, y, por otra, presenta desde la visión de los estudiantes algunas diferencias entre el aula de clase tradicional y la comunidad de investigación.

La posibilidad de enseñar filosofía desde el grado sexto, y al mismo tiempo reorientar su función en los grados décimo y once, coincide con el proceso de resignificación del Proyecto Educativo Institucional en términos de pertinencia, calidad y acceso a la educación; una propuesta educativa acorde con las expectativas de las comunidades frente al proceso educativo, donde el enfoque pedagógico permita elevar las posibilidades de acceso a la educación técnica, tecnológica y universitaria, con el fin de incidir no solo en el mejoramiento de la calidad educativa en la región,

6 Los estudiantes del CJC son niños y jóvenes del sector rural provenientes de once municipios de las provincias de Sugamuxi y Tundama; ellos viven en el internado del colegio, allí pasan la gran parte de su tiempo; los fines de semana o en periodos de vacaciones viajan a sus municipios a estar con sus familias. El tiempo en el internado lo dividen entre las jornadas de clase, las actividades en la granja didáctica y productiva y compromisos con el bienestar del internado. 
sino también en la calidad de vida de los estudiantes y sus comunidades. En este escenario, y a partir del proceso de investigación, se revisaron entonces, los fundamentos de la propuesta de $\mathrm{FpN}$; de ella se adoptaron la comunidad de investigación, como posibilidad de transformar los modos y las formas del aula de clase tradicional; la discusión filosófica, como medio para desarrollar destrezas y habilidades de pensamiento; sus novelas, como ejemplo metodológico para el trabajo sobre las preguntas e inquietudes de los estudiantes, y el objetivo de lograr que los niños y jóvenes puedan pensar por sí mismos como esencia del proyecto en la institución educativa.

La iniciativa de enseñar filosofía bajo los fundamentos anteriormente señalados tropieza en sus inicios con diversos obstáculos relacionados con la manera como se había presentado la asignatura a profesores y estudiantes. Las percepciones eran consecuencia de ocho años de experiencia institucional con el Sistema Aprendizaje Tutorial, $\mathrm{SAT}^{7}$, donde la clase de filosofía se orientaba por medio del desarrollo de módulos que contenían para cada capítulo una serie de actividades que el estudiante resolvía según su criterio. Se consideraba que los módulos contenían lo necesario para el proceso de aprendizaje del estudiante en la asignatura, razón por la cual no había docente en el área, sino un grupo de tutores que orientaban diferentes grupos y de diferentes asignaturas a la vez. Respecto a la enseñanza de la filosofía, los tutores tenían la función de revisar y valorar el desarrollo de los ejercicios propuestos para cada actividad.

Bajo este sistema, el objetivo institucional consistía en reafirmar la condición campesina de los estudiantes por medio de la capacitación en proyectos productivos que les permitiera generar ingresos para mejorar su bienestar y el de su familia. Al no encontrar relación entre lo que se hacía en la asignatura y el objetivo institucional, los estudiantes consideraban que la función de la enseñanza de la filosofía en la institución educativa consistía en la preparación para las pruebas ICFES. Cabe señalar que aún después de iniciar con el proceso de transformación del aula de clase

7 La finalidad de la metodología del Sistema de Aprendizaje (SAT) estaba dirigida a desarrollar, de manera práctica y vivencial, competencias y habilidades que permitieran al estudiante formular proyectos para el beneficio del desarrollo cultural y económico de sus comunidades. Bajo este objetivo, los procesos de enseñanza y de aprendizaje estaban orientados a desarrollar cinco capacidades: científica, matemática, lenguaje y comunicación, tecnología y servicio a la comunidad. Aun cuando el proceso fue orientado al desarrollo de áreas básicas, los estudiantes debían responder a preguntas especificas de química, filosofía, física e inglés en pruebas externas, situación que incido en la revisión del Proyecto Educativo Institucional en el año 2007. 
tradicional, los estudiantes reconocían como objetivo de la iniciativa institucional la intención de mejorar los puntajes de la asignatura en esta prueba. Estudiante de grado once:

Considero que se dicta desde el grado sexto, ya que, por ejemplo, si la comienzan a dictar desde el grado décimo la información que vamos tener en el grado once para presentar la prueba ICFES va a ser muy cortica, entonces si nuestra formación comienza desde el grado sexto vamos a tener varios conocimientos para estar bien preparados en nuestra prueba.

En esta percepción, la enseñanza de la filosofía corresponde con los supuestos de la educación tradicional señalados en apartados anteriores. Pareciera que la única y posible utilidad de la enseñanza de la filosofía está puesta en la preparación para pruebas externas. Sin embargo, el problema no radica en la preparación para asumir la prueba, sino en el hecho de relacionar el tiempo de las clases con la cantidad de información que se puede memorizar. Por otra parte, la palabra dictar implica que la información la tiene el docente, y a mayor información transmitida, mayor conocimiento para asumir la prueba. Esta visión sobre la enseñanza de la filosofía difiere notablemente del punto de vista expresado por un estudiante de grado sexto, que participa en la comunidad de investigación:

Se dicta desde el grado sexto porque allí exploramos cómo se hace una pregunta, por qué y cómo se hace una pregunta, por qué la debemos hacer, qué tipos de preguntas hay y cómo dar razones que justifiquen todo lo que decimos... la filosofía nos enseña a preguntar más y más.

Aun cuando aquí persiste la palabra dictar, se describen otras acciones que, a diferencia de las señaladas por la alumna de grado once, pueden ser parte de la práctica crítica de la educación y, con ello, parte de otras formas de ver la enseñanza de la filosofía. El valor de la pregunta, que reconoce el estudiante, representa el trabajo de la comunidad de investigación y el sentido de la discusión filosófica en la cotidianidad. Así lo expresa un estudiante del grado séptimo: “...la filosofía nos sirve para solucionar un problema que haya en la casa, solucionar pidiendo la palabra y hablando, razonando sobre las preguntas y lo que decimos y sobre lo que dicen y preguntan nuestros familiares para poder solucionar el problema". Ver la enseñanza de la filosofía como preparación para el ICFES dista mucho de reconocer en ella un ejercicio de discusión que beneficia de alguna manera la forma como los estudiantes logran analizar, asumir y resolver problemas. Este puede ser el beneficio más valioso que un estudiante 
pueda obtener de la comunidad de investigación, y, tal vez, el mejor que la enseñanza de la filosofía puede brindarle.

Estudiante de grado octavo:

La filosofía es una forma de vida, de pensar racional y de poder reflexionar sobre las situaciones que se le presentan... también la filosofía nos ayuda cuando se nos presenta una situación de debate, nos puede ayudar a defender ideas, exponerlas y también nos ayuda a ser críticos, personas que pueden pensar por sí mismas, no en base a lo que piensen los demás.

Cuando los objetivos no se imponen, es posible que cada miembro de la comunidad de investigación configure sus propios objetivos, platee sus propias metas y busque oportunidades que le permitan alcanzarlas. La participación en principio fue de socialización de experiencias de la vida cotidiana, podrían pasar una o dos horas, y los estudiantes escuchaban atentos historias de reencuentros familiares, apariciones de brujas y duendes, tomas y hostigamientos guerrilleros y, sobre todo, anécdotas de la vida en el campo. En ocasiones, cuando el narrador perdía el hilo, algunos estudiantes hacían preguntas precisas; en otras, cuando se dejaba llevar por la fantasía, surgían algunos cuestionamientos que contrastaban con lo que posiblemente no era real para ellos. La comunidad de investigación no deja atrás el trabajo sobre la experiencia del estudiante, por el contrario, toma las inquietudes y preguntas que surgen allí para asegurar la relación y utilidad de la discusión filosófica en la manera como el estudiante pueda asumir sus problemas y los de su comunidad.

El valor de la comunidad de investigación depende del éxito que logran los estudiantes en la discusión filosófica. En cada una de las sesiones existe una serie de acciones que bajo la revisión continua del grupo y la autocorrección del estudiante terminan influyendo en el mejoramiento de la calidad académica. Al igual que en la comunidad de investigación, en otros escenarios académicos, y donde el docente lo promueva, los estudiantes trabajan sobre las perspectivas de los demás, construyen puntos de vista en conjunto, evalúan y analizan los argumentos que justifican una intervención, hacen preguntas y buscan alternativas de análisis y solución a las problemáticas en discusión. Así lo manifiesta la profesora de inglés de la institución:

El trabajo de la comunidad de investigación se ve reflejado en la participación que hacen los estudiantes en diferentes espacios; ellos se interesan por aquellos temas que los afectan, leen y se enteran 
sobre las problemáticas, y después participan en discusiones sobre el tema y dan argumentos que justifican lo que dicen. En los espacios de diálogo y discusión de inglés, los estudiantes hacen relaciones que generan preguntas...

El trabajo de la comunidad de investigación ha permitido que los estudiantes, que en principio evitaban participar por diferentes circunstancias, se motiven a expresar y compartir con otros sus puntos de vista y perspectivas sobre las situaciones que les generan preguntas e inquietudes. El ejercicio de pensar por sí mismo con otros influye en la posibilidad de llevar intervenciones que narran situaciones o historias a intervenciones que generan cuestionamiento, preguntas, argumentos y, sobre todo, revisión y análisis de lo que se dice, piensa y hace. Esta forma de trabajo que no está determinada bajo una estructura fija, genera actitudes y hábitos que benefician en parte las actividades propias de otras asignaturas. En palabras de la profesora del área de emprendimiento y tecnología,

La comunidad de investigación hace un aporte realmente importante al área de emprendimiento y tecnología; tenemos estudiantes que formulan proyectos tanto en la parte productiva como otros que aplican a la parte académica; los estudiantes han desarrollado una capacidad de leer su contexto, de formularse preguntas a raíz de diversos problemas que ellos ven allí, y llevarlas al aula para generar discusión con sus compañeros.

Así, la transformación del aula de clase tradicional trasciende al ejercicio de discusión, diálogo y conversación que se genera en otras asignaturas. De tal manera que no es posible hablar de una, sino de varias comunidades de investigación comprometidas con la indagación, el cuestionamiento y la reflexión crítica. Hablar de la posibilidad de hacer discusión filosófica en clases diferentes a las de filosofía no implica que los docentes y estudiantes traten problemas propios de la historia de filosofía, sino más bien, que discutan los temas de su interés bajo ciertas condiciones de rigurosidad. Allí, al igual que en la clase de filosofía, los estudiantes pueden dar y solicitar razones, analizar y estudiar la validez de los argumentos, contemplar alternativas de análisis y solución, y, sobre todo, problematizar todo aquello que se presenta como verdad irrefutable. En este sentido, la discusión que se genera en la comunidad de investigación se convierte en una fuerte estrategia para generar la reflexión sobre el propio pensamiento y sobre las formas de razonar. Acciones que se convierten en directrices de un proyecto transversal dirigido a lograr que niños y jóvenes de diferentes edades puedan asumir una actitud crítica y 
creativa no solo frente a la discusión que se genera en la comunidad de investigación, sino también frente a las problemáticas que se presentan en su vida cotidiana. Una actitud que problematiza lo que para otros pareciera ser evidente, que no se queda en el cuestionamiento ni instaura verdades irrefutables, sino que, más bien, va más allá de lo que piensa tradicionalmente, contemplando otras alternativas de análisis y solución. Estas y no otras acciones permitirán que niños y jóvenes de diferentes edades puedan pensar por sí mismo, pensar de forma crítica y creativa.

\section{A manera de cierre}

Enseñar filosofía en la educación básica y media se corresponde con la función crítica y creativa de la filosofía, que orienta, por una parte, la reflexión sobre la práctica docente, y, por otra, la revisión de algunas propuestas educativas. Un docente, además de cuestionar y oponerse justificadamente a ciertas maneras de ver y enseñar la filosofía, también propone acciones innovadoras dirigidas a generar otras condiciones de posibilidad para la enseñanza de la filosofía, donde los estudiantes encuentren un marco de referencia que dé sentido a las preguntas e inquietudes que surgen en su experiencia.

La enseñanza de la filosofía puede ser útil en el proyecto de trasformación de la sociedad, en la medida en que logra trascender el aula de clase; por esta razón, el trabajo de la comunidad de investigación no consiste en suscitar una discusión afanosa y desinteresada que poco o nada incide en el mejoramiento del pensamiento y la comunicación, sino en buscar que los estudiantes se preocupen por sí mismos, por la forma en que razonan y la manera como resuelven los problemas que se presentan en su cotidianidad.

$\mathrm{Al}$ trabajar con las inquietudes y preguntas que se generan de la experiencia del estudiante, los alcances de la discusión filosófica no solo se observan en la manera como los estudiantes abordan el debate en la comunidad de investigación, sino también en otras situaciones donde deben asumir una posición crítica que les permita participar de forma activa en el análisis y solución de diferentes problemáticas. Por esta razón, los docentes que adoptan los modos y formas de acción de la comunidad de investigación logran priorizar el debate, el diálogo y la discusión sobre los contenidos específicos de cada área, sobre la relación de los temas de la clase con la vida del estudiante y sobre su utilidad para resolver dificultades y problemáticas del entorno. 
El ser el asombro el origen de la filosofía implica que esta sea una actividad racional que parte de lo dado, es decir, de la experiencia, de lo que le pasa a un individuo de forma personal e irrepetible. Al desconocer la experiencia, los docentes terminan por ignorar los presaberes y, con ello, las preguntas e inquietudes de los estudiantes, convirtiendo las clases de filosofía en un espacio para revisar, muchas veces de forma desfragmenta, la historia de la filosofía. En este sentido, los docentes tienen la responsabilidad de relacionar y conectar en la discusión filosófica lo que pasa con el estudiante en su vida cotidiana con el inmenso caudal de la tradición filosófica.

Al respecto, la reflexión sobre la práctica docente debe conducir a transformar lo que se hace en el aula de clase, los materiales que se utilizan y los objetivos que se persiguen a nivel institucional con la enseñanza de la filosofía. En el caso de las instituciones educativas donde la enseñanza de la filosofía es solo para los grados décimo y once, la posibilidad de transformación implica la crítica a esas pretensiones que posicionan la utilidad de la filosofía como preparación para pruebas externas, y en el caso de la básica media, el cuestionamiento a esas afirmaciones que califican a niños y jóvenes como sujetos incapaces de pensar por sí mismos, dependientes de la sabiduría de sus docentes. De este modo, las propuestas de innovación pedagógica referidas a la enseñanza de la filosofía deben generar análisis y evaluación sobre los valores, las creencias y los saberes que revisten la función del docente y del estudiante en los procesos de enseñanza y de aprendizaje; acciones que atiendan a la posibilidad de pensar y concebir desde otros puntos de vista la función de la filosofía, la infancia y, por supuesto, el papel del docente.

\section{Referencias}

García, F. (2006). Pregunto, dialogo, aprendo. Cómo bacer filosofía en el aula. Madrid: Ediciones de la Torre.

Kohan, W. y Waskman, V. (2000). Filosofía para niños. Discusiones y propuestas. México: Ediciones Novedades Educativas.

LaGo, C. (2006). Redescribiendo la comunidad de investigación. Pensamiento complejo y exclusión social. Madrid: Ediciones de la Torre.

Lipman, M. (2002). La filosofía en el aula. Madrid: Ediciones de la Torre.

Lipman, M. (1998). Pensamiento complejo y educación. Madrid: Ediciones de la Torre.

López, F. (2005). Filosofía en la escuela. La práctica de pensar en las aulas. España: Grao.

Splintter, L. y SHarp, A. (1995). La otra educación. Filosofía para niños y la comunidad de investigación. Argentina: Manantial. 